
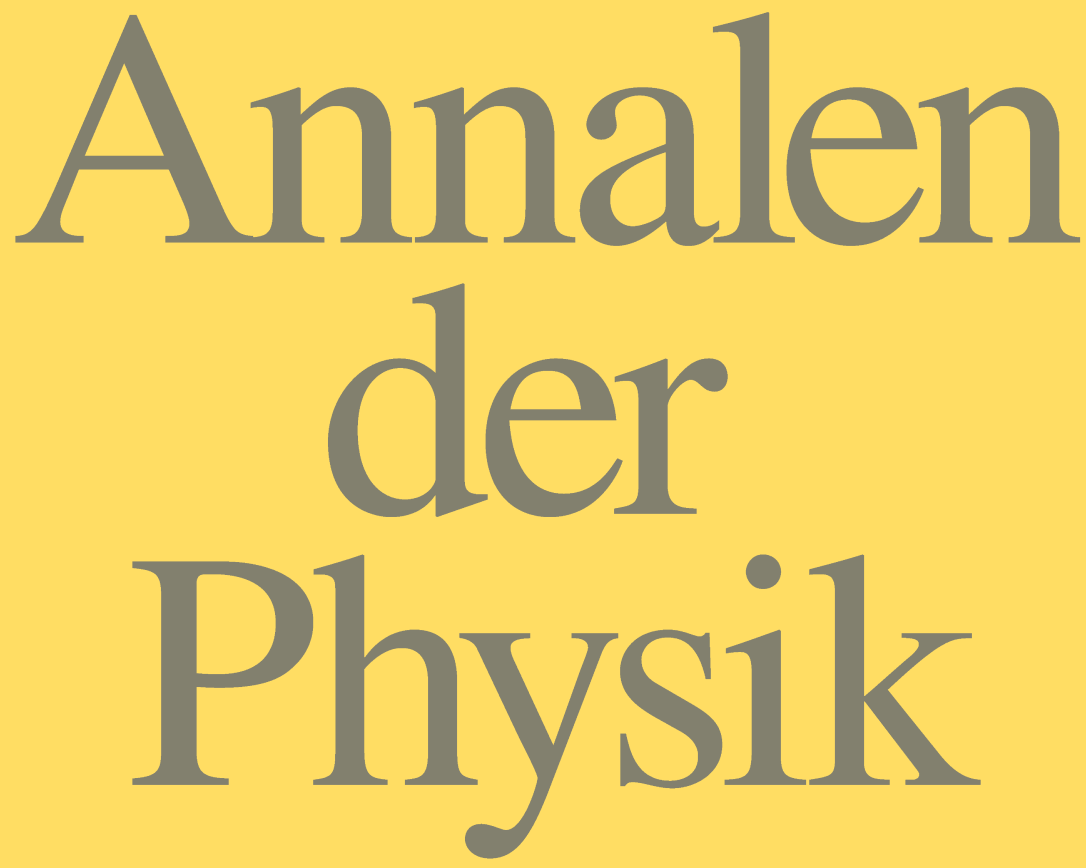

Founded in 1790

Editor-in-Chief

U. Eckern

Augsburg

Editors

F. W. Hehl

Köln
B. Kramer

Bremen
G. Röpke

Rostock
A. Wipf

Jena

(\$)WILEY-VCH

www.ann-phys.org 


\title{
Quantum mechanics needs no consciousness
}

\author{
Shan Yu ${ }^{1, *}$ and Danko Nikolić ${ }^{1,2, * *}$ \\ ${ }^{1}$ Department of Neurophysiology, Max Planck Institute for Brain Research, Deutschordenstr. 46, \\ 60528 Frankfurt am Main, Germany \\ ${ }^{2}$ Frankfurt Institute for Advanced Studies, Johann Wolfgang Goethe University, Ruth-Moufang-Str. 1, \\ 60438 Frankfurt am Main, Germany
}

Received 19 April 2011, revised 12 September 2011, accepted 16 September 2011 by U. Eckern Published online 13 October 2011

Key words Measurement problem, wave function collapse, observer, double-slit experiment.

It has been suggested that consciousness plays an important role in quantum mechanics as it is necessary for the collapse of wave function during the measurement. Here we formulated several predictions that follow from this hypothetical relationship and that can be empirically tested. Experimental results that are already available suggest falsification of these predictions. Thus, the suggested link between human consciousness and collapse of wave function does not seem viable. We discuss the implications of these conclusions on the role of the human observer for quantum mechanics and on the role of quantum mechanics for the observer's consciousness.

\section{Introduction}

The nature of human consciousness and its relation to the physical reality is one of the most puzzling fundamental issues about ourselves and about our interaction with the world we live in. An interesting proposal has been put forward of a link between the seemingly distant quantum mechanics and consciousness, leading to a direct, yet bizarre bridge between the mental and the physical. Specifically, it has been suggested that the consciousness plays a vital role for the quantum mechanics as it is capable of, and even required for affecting physical events through the collapse of the wave function.

According to most interpretations of the quantum mechanics (with the exception of the hidden variable theory, e.g., [1]), a quantum system can take two, very different forms: one is continuous and deterministic, and is described by a wave function [2] that represents a superposition state; and the other is discontinuous and stochastic, because the system "jumps" (collapses) suddenly during the measurement from a superposition state into a single randomly-chosen eigenstate. The question about how and why such a collapse occurs is usually referred to as the measurement problem.

Von Neumann [3] was among the first people to suggest a unique relation between the human observer and the measurement problem (for related reviews, see [4-6]). According to him, the measurement process consists of three main components: the system to be observed (S), the measuring instruments (M) and the observer (O). In order to measure the state of S, a physical device is needed. Let us denote it as M1. This device has also its own states (e.g., the positions of the hand of a gauge) but these are sensitive to, and interact with $\mathrm{S}$. The problem becomes more interesting when one realizes that, as S and M1 interact they form a combined system $\left(\mathrm{S}^{\prime}\right)$ that also needs to be observed. This observation can be made only by another

\footnotetext{
Corresponding author E-mail: yushan.mail @gmail.com

Current address: Section on Critical Brain Dynamics, National Institute of Mental Health, 9000 Rockville Pike, Bethesda, MD 20892, USA

** E-mail: danko.nikolic@ googlemail.com
} 
measuring device (M2), but then again $\mathrm{S}^{\prime}$ in combination with $\mathrm{M} 2$ forms $\mathrm{S}^{\prime \prime}$, which needs yet another measuring device M3, and the chain can go on up to the infinity. According to von Neumann, any measuring instrument, $\mathrm{M}$, although a macroscopic object, should obey the fundamental rules of quantum mechanics, much like $\mathrm{S}$. Thus, before the state of a device $\mathrm{M}$ has been measured, the device must be in a superposition state, and this holds for every device in the chain, i.e., M1, M2, etc. This property postpones iteratively the collapse of the wave function to an ever later measuring device positioned higher and higher on the hierarchy, rendering thus the problem unsolvable. Von Neumann reasoned that in order to break this infinite chain of measurements and to give to the whole process a superposition-free, definite end, something with a very distinct property - that cannot be described by the above procedure and hence, by the quantum mechanics - needs to be involved. He suggested, although only implicitly, that the "subjective perception" of the human observer, O, or its "abstract ego" plays this important chain-braking role [3]. Shortly after this implicit proposal, London and Bauer [7] suggested explicitly that the collapse of the wave function and thus, the measurement of a quantum process, cannot occur without a registration of the results in the observer's consciousness. This new role of human consciousness in theoretical physics was defended by pointing out that consciousness has a "completely special character", which is "the faculty of introspection", and which in turn allows a person to be aware of the status of its own awareness [7], corresponding to the measurement of itself and abrogating thus the need for any additional measurement device. Therefore, the registration of a result in consciousness brings ultimately the initial system of the measurement into a new form - taking a single eigenstate. Later, Wigner popularized this link between consciousness and collapse of wave function passionately [5]. Wigner suggested that "It is the entering of an impression into our consciousness which alters the wave function.", and "It is at this point that consciousness enters the theory unavoidably and unalterably." (cited from [8]). Importantly, however, Wigner dropped this opinion completely at his final years [5].

Critical evaluation and heated debate on this hypothesis has not been missing (e.g., [4-6, 8-19]. Many of those studies address this issue from the philosophical point of view. Although they went to deep and interesting levels and brought up exciting ideas about fundamental aspects of the relationship between the human mind and the physical world, those profound analyses did not reach a simple and clear conclusion that would be widely accepted. Partly due to this reason, the hypothesis that consciousness causes (or at least correlates with) the collapse of the wave function and, therefore, may play an important role in the quantum mechanics remained a theoretical possibility. Although not preferred by most physicists, this solution to the measurement problem is still strongly suggested even in some recent works (e.g., [12, 17]).

In the present paper, we do not aim to provide another philosophical argument. Instead, we attempt to address this issue from an empirical perspective. We reformulate von Neumann's hypothesis as an empirically testable problem. We then attempt to falsify the hypothesis on the basis of the existing empirical evidence, as already suggested elsewhere $[11,13,20]$. In addition, we identify the experiments that need to be made in order to test the hypothesis more thoroughly.

\section{Experimental design}

First, let us formulate the hypothesis to be tested:

The event of forming, in the observer's mind, an explicit phenomenal representation of the result of a quantum measurement is necessary for the wave function (superposition state) of the system to collapse into a single eigenstate.

This hypothesis reflects the suggested role of consciousness in collapsing the wave function, as we introduced in the previous section. By using the logical symbol of implication $(\Rightarrow)$ we can write this hypothesis formally as:

$$
\mathrm{CWF} \Rightarrow \mathrm{PR},
$$


where CWF stands for "collapse of wave function" and PR for "phenomenal representation", meaning that the collapse of wave function should be always associated with a corresponding event of registering the results of a measurement in consciousness. Or, by using a logical negation $(\neg)$ we can express this hypothesis equivalently as a contraposition of (1):

$$
\neg \mathrm{PR} \Rightarrow \neg \mathrm{CWF},
$$

meaning that the collapse of wave function should never occur if the corresponding result of measurement has not been registered by a conscious observer. We emphasize that relations (1) and (2) are mathematically equivalent and represent accurately the critical role of consciousness in collapsing the wave function, as expressed in our hypothesis.

There are multiple definitions of consciousness, each serving a different purpose [10]. The present discussion is concerned with the subjective phenomenal representations in the mind of an individual observer. But then there is also a need to measure the presences of such subjective experiences. To this end, we suggest to adopt a commonly used operationalization of awareness that is based on appropriate verbal reports (see e.g., [21,22]). For example, one may report: "The light beam hit the screen on the left side.", "The oscilloscope showed $1 \mathrm{MHz}$ signal." or "The gauge pointed to $5 \mathrm{mV}$ ". Verbal reports do not cover comprehensively all possible aspects of conscious experiences. Nevertheless, this operationalization is sufficient for the current analyses, and is fundamentally consistent with the general concept of consciousness outlined in the introduction.

It is clear that this hypothesis and equivalently, the relations (1) and (2), cannot ever be proven true, much like any other theoretical statement in science cannot ever be proven true [23]. However, the hypothesis can be proven untrue simply by finding a counter example, that is, by finding an experimental setup in which the collapse of wave function is dissociated from a conscious representation of the outcome of a measurement. Thus, the first goal is to find an experimental setup that would allow one to assess both the state of consciousness and, independently, the state of the wave function. To this end, we consider an adapted version of the experimental setup used by Kim et al. [24]. This kind of experiment was originally proposed by Scully and Drühl [25] to acquire "which-path" information of a photon in a double-slit experiment (see Fig. 1). First, one photon is generated in the pump and travels through the double-slit. This photon can hit either region A or B, which are located on the nonlinear optical crystal in order to produce an entangled pair of photons. In the resulting pair, one photon (the signal) travels through the lens LS and is detected by the detector $\mathrm{D}_{0}$ positioned at the focal plane of LS. The other photon (the idler) is routed the other direction and travels through a prism to be directed either towards $\mathrm{D}_{1}$ or $\mathrm{D}_{2}$, depending on the region in which it has been initially produced (A or B). Thus, by knowing which of the two detectors has been hit by the idler photon, we know which path the original photon - the one that travels through the double-slit - has taken.

Next, we analyze the system more closely. Assume first that the laser emits only one photon at a time. The state of the photon, $\Psi$, can be described as:

$$
\Psi=(1 / \sqrt{ } 2)(|\mathrm{L}\rangle+|\mathrm{R}\rangle)
$$

where $|\mathrm{L}\rangle$ and $|\mathrm{R}\rangle$ indicate the photon's states, i.e., whether the photon passed through the left or right slit, respectively. As a result, after the generation of a photon pair in the optical crystal, the signal photon may take either both paths (1 and 2) simultaneously (if the pair is in a superposition state, $\left|1,1^{\prime}\right\rangle+\left|2,2^{\prime}\right\rangle$, and hence the wave function did not collapse) or through only one of the two (if the pair is in a single state, $\left|1,1^{\prime}\right\rangle$ or $\left|2,2^{\prime}\right\rangle$ due to a collapse of the wave function). If the photons are always in a superposition state, after a sufficient number of photons have been registered at $\mathrm{D}_{0}$, the distribution of the registered locations along the $x$-direction will exhibit the standard Young's double-slit interference pattern, which is manifested by a distribution consisting of a series of peaks and troughs [24]. In contrast, photons that assume a single state will not produce such an interference pattern but will instead form a single-peaked distribution [24]. Thus, the presence of the interference pattern at $\mathrm{D}_{0}$ indicates whether the wave function of 


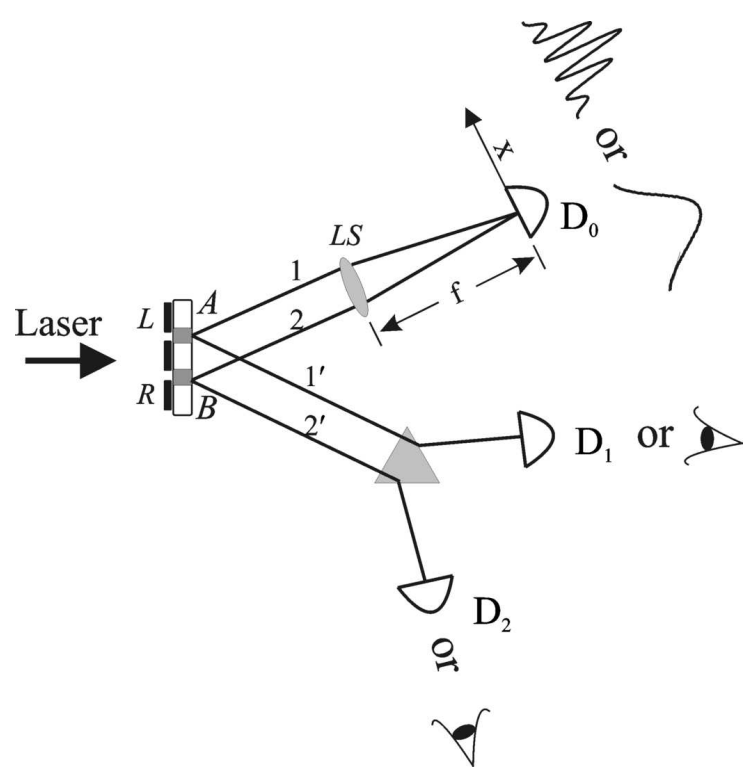

Fig. 1 The proposed experimental setup that can be used to test whether collapse of wave function and consciousness about the outcome of a measurement are dissociated. This double-slit experiment is a modification of an actual experiment that has been carried out [24]. See the main text for details.

the original photon collapsed or not ${ }^{1}$. Hence, regarding the collapse discussed here, the relevant information (corresponding to the results of a measurement) is which path (i.e., $L$ or $R$ ) the photons took. Now, we can derive the predictions for this experimental setup that follow from the previously formulated relation (2):

The interference pattern should be visible if "which-path" information has not been registered in consciousness of the observer (i.e., the experimenter).

Specifically, we expect to find an interference pattern at $\mathrm{D}_{0}$ in the following conditions:

i) No actual attempt to measure the "which-path" information was made, that is, $\mathrm{D}_{1}$ and $\mathrm{D}_{2}$ are not implemented at all.

ii) The "which-path" information was measured as $D_{1}$ and $D_{2}$ are implemented in order to interact with the incoming photons. However, no results were recorded by a macroscopic device and hence are not visible or accessible to a human observer in any way ${ }^{2}$.

iii) The "which-path" information was measured by a macroscopic device such as $\mathrm{D}_{1}$ and $\mathrm{D}_{2}$. The results were not recorded but were instead presented to a human observer temporarily and directly such that the relevant information entered the sensory system but, at the same time, the observer was distracted in order to prevent conscious detection of this event. In other words, the information necessary to achieve phenomenal representation was available in the nervous system, but conscious phenomenal experience was actually not realized solely due to internal psychological processes. Thus, there were only non-phenomenal (non-conscious) mental representations.

The latter experiment can be implemented by presenting the relevant information through a memory-less device (e.g., an old fashion gauge-based instrument) or by feeding the idle photons directly into the retina (or after an amplification) and, thus, having human eyes serve directly the function of $\mathrm{D}_{1}$ and $\mathrm{D}_{2}$ (e.g., see $[6,26-28])$. A distraction that prevents one from consciously detecting a stimulus is made routinely in psychological studies and can be achieved by various means, such as the visual masking [29], attentional blink [30], binocular rivalry [15], change blindness [31], execution of a concurrent tasks [21], or simply by cluttering the visual scene [32]. The interference with conscious perception can be made even more

1 Interference pattern is not necessarily obvious from the entire distribution. So proper separation of sub-populations of registered photons may be needed [24].

2 Tests similar to conditions i) and ii), but not for studying the role of consciousness, were suggested previously (Ross Rhodes, www.bottomlayer.com). 
directly by using trans-cranial magnetic stimulation (e.g., [22]). One could apply a magnetic pulse above e.g., visual cortex, in order to interrupt the information processing in this brain region, preventing hence conscious perception of the visual stimuli.

Moreover, by using the aforementioned methods, one can manipulate gradually the level of subjective certainty with which the observer judges this information to be present. This should then, according to the hypothesis, affect the contrast of the interference pattern accordingly - as it has been shown in physical experiments by manipulating the extent to which the "which-path" information was available, e.g., by changing the position of photon detector [20] or by attenuating the optical transmission [11].

A verification of these three predictions through empirical tests we propose to be a necessary requirement to warrant the hypothesis that consciousness of the outcome of a measurement is necessary for the wave function to collapse. By formulating these predictions we make this hypothesis empirically testable and hence, falsifiable.

\section{Existing evidence}

The experimental results that falsify predictions i) and ii) already exist. Firstly, in experiments similar to that proposed here (e.g., $[11,20,33])$, it was shown that if "which-path" information was in principle obtainable, then even though no actual attempt was made to extract this information (i.e., to measure it), no interference pattern was found. For example, in the experiment carried out by Zou et al. [33], the interference pattern formed by the signal photons could only be observed when the paths of idler photons were aligned, i.e., the "which-path" information was destroyed. If the idlers were misaligned to allow the source of the signal photons to become distinguishable, the interference pattern disappeared. Interestingly, under such conditions, it is not important whether a detector is actually in place ready to make the measurement of "which-path" or not. As long as such measurement could be made, i.e., the photon path is in principle identifiable, the interference is wiped out [33]. Thus, the first prediction of consciousness hypothesis is false.

Secondly, in another set of experiments, "which-path" information was measured but was not recorded by a macroscopic device and, therefore, was not accessible to a conscious observer. Under such conditions, also no interference pattern was found. For example, in the experiments reported by Eichmann et al. [34] and Dürr et al. [35], the "which-path" information was only stored in the state of a single atom. Results demonstrated unambiguously that even if such microscopically stored information was not actually read out, the mere fact that it could be read out ensured the absence of the interference pattern. Therefore, the existing evidence indicates that the second prediction is also false.

To the best of our knowledge, no direct attempt was made to test the third prediction. However, the expectations for such experiment are clearly set by the evidence related to predictions i) and ii). That is, if no interference pattern was obtained when the "which-path" information was not fed into the eye of the observer (e.g., carried by the idler photon as illustrated in Fig. 1), the same is expected to occur if the photon reached the observer's retina but the person was distracted and hence, not able to detect the event.

Taken together, the existing experiments suggest clear conclusions regarding the predictions we derived. All predictions have been falsified. The existence of interference patterns depends solely on whether the "which-path" information is in principle obtainable [11,20,33-35]. Whether such information is registered in consciousness of a human observer, one can conclude, is irrelevant. Consequently, this conclusion leaves no other option but to reject the collapse-by-consciousness hypothesis.

\section{Discussion}

We first formulated a hypothesis about the relationship between the collapse of the wave function and conscious perception. Our subsequent analyses lead to the conclusion that this hypothesis is already falsified 
by the existing empirical results, which forced us to conclude the following: Conscious access to the information about the outcome of a measurement is not necessary for the collapse of the wave function - a conclusion similar to those suggested elsewhere $[11,13,20]$.

Do the present analyses really tell us something about the relation between consciousness and quantum mechanics? One may argue that with the current experimental setup (shown in Fig. 1), present-day quantum mechanics can predict, correctly, no interference pattern in $\mathrm{D}_{0}$. This seems to hold irrespective of what happens with the idler photons (except for "erasing" the "which-path" information that is carried by those photons, see [24]), and certainly irrespective of whether a conscious observer is involved and where the attention of this observer is directed to. Therefore, this setup cannot tell us anything new about the relation between consciousness and quantum mechanics that we did not know before.

According to this assertion, a really interesting test would involve some observables not determined by the current quantum theory. Only in that case, consciousness of the observer would be given a chance to affect the results and only in that case would we have a possibility to search empirically for novel discoveries. However, designing such an experiment would require finding a situation in which the present-day quantum mechanics is incomplete (e.g., the current theory does not predict whether interference will be observed) or incorrect (e.g., the current theory makes incorrect prediction about the interference pattern). Although such possibility cannot be fully excluded, given the overwhelming evidence supporting the correctness and completeness of the quantum mechanics (e.g., through the violation of Bell's inequality), we believe that the existing theory may have not left any space for adding the observer's consciousness as an additional variable that would affect the experimental result.

This conclusion casts strong doubts on those interpretations of quantum mechanics that place the observer's mind in a special position. For example, the many-minds interpretation [36] suggests that the single state revealed after the measurement is only perceived by the mind and does not reflect any physical event outside this particular mind. The current analysis renders such no-collapse-outside-the-mind interpretations untenable. More generally, since consciousness does not play a vital role in the measuring procedure, our conclusion supports the previous suggestion that the quantum mechanics does not assign human observer a more special role than it has been assigned by the classical theory [8]. Note that here we do not intend to describe the exact nature of this role. We only suggest that, irrespective of what this role actually is, there are no differences between quantum and classical physics.

Does consciousness need quantum mechanics? Our analysis also shed a new light on a related, interesting proposal for understanding the nature of consciousness. The assumption that the mind has a special property and can, through collapse, perceive a single state has led to a symmetrical proposal that the collapse of the wave function - within the brain - is responsible for the emergence of consciousness (e.g., see [37]). That is, consciousness would be simply the ability to perceive a single state out of the superposition states. This idea is explicitly expressed in e.g. [37] but is implicitly assumed in other mind-centered interpretations of the quantum mechanics [36]. Although the idea is fascinating as it promises to explain two deep mysteries - the collapse of wave function and the nature of consciousness - at the same time, our analysis provides strong reasons for refuting it.

It is helpful to note that the current analysis is aimed towards clarifying a specific relationship between the mind and the physical world, namely towards the hypothetical necessity of conscious registration of a measurement result to induce a collapse of the wave function. We do not try to draw any general conclusions about the relation between physical reality and mental phenomenal representations. Also, the current analysis does not support or disprove directly any other specific suggestions related to 1) applying the quantum theory to explain neural activities in the brain, and 2) extending the conceptual features of the quantum theory onto studies of psychological and cognitive phenomena, as those suggestions do not necessarily depend on the collapse-by-consciousness assumption. For an informative review of these works, see [38].

In conclusion, the available evidence does not indicate that the observer's explicit phenomenal representation about the outcome of a measurement plays a role in collapsing the wave function. Thus, the idea that by mere observation the experimenter creates physical reality does not seem viable. This supports Wigner's 
opinion in his later years and promises to fulfill his hopes - that we "will not embrace solipsism" and "will let us admit that the world really exists" (cited from [4]). Perhaps equally importantly, we can add our own hope that the rejection of the role of consciousness in quantum mechanics will also lead us to re-evaluate the proposals that quantum mechanics is vital for explaining the consciousness. Having these two deep mysteries disentangled one from the other might be an important step forward towards understanding each of them.

Acknowledgements We thank Thomas Metzinger, Markus Arndt, Anton Zeilinger, Rajarshi Roy and Nick Herbert for helpful comments. We are especially grateful to Hrvoje Nikolić for the fruitful discussions at the early stages of this project.

\section{References}

[1] D. Bohm, Phys. Rev. 85, 166-193 (1952).

[2] E. Schrödinger, Phys. Rev. 28, 1049-1070 (1926).

[3] J. von Neumann, Mathematical Foundations of Quantum Mechanics, Chap. VI, (1932) translated by R. T. Beyer (Princeton University Press, Princeton, 1955).

[4] H. Primas and M. Esfeld, PhilSci Archive (1997), http://philsci-archive.pitt.edu/1574.

[5] M. Esfeld, Stud. Hist. Philos. Sci. B 30, 145-154 (1999).

[6] F. H. Thaheld, BioSystems 81, 113-124 (2005).

[7] F. London and E. Bauer, La théorie de l'observation en mécanique quantique (1939), English translation in Quantum Theory and Measurement, edited by J. A. Wheeler and W. H. Zurek (Princeton University, Princeton, 1983) p. 217-259.

[8] A. Shimony, Am. J. Phys. 31, 755-773 (1963).

[9] J. G. Cramer, Rev. Mod. Phys. 58, 647-687 (1986).

[10] D. J. Chalmers, The Conscious Mind: In Search of a Fundamental Theory, Chap. 10 (Oxford Univ. Press, New York, 1996).

[11] L. Mandel, Rev. Mod. Phys. 71, S274-S282 (1999).

[12] M. B. Menskii, Phys.-Usp. 43, 585-600 (2000).

[13] C. Brukner and A. Zeilinger, Philos. Trans. R. Soc. Lond. A, Math. Phys. Eng. Sci. 360, 1061-1069 (2002).

[14] S. French, Stud. Hist. Philos. Sci. B 33, 467-491 (2002).

[15] C. Koch and K. Hepp, Nature 440, 611-611 (2006).

[16] R. Penrose, The Road to Reality: A Complete Guide to the Laws of the Universe, Chap. 29 (Vintage Books, London, 2005).

[17] B. Rosenblum and F. Kuttner, Quantum Enigma: Physics Encounters Consciousness, Chap. 16 (Oxford Univ. Press, New York, 2006).

[18] M. Nauenberg, Found. Phys. 37, 1612-1627 (2007).

[19] H. P. Stapp, Mindful Universe: Quantum Mechanics and the Participating Observer, Chap. 12 (Springer, Berlin, 2007).

[20] A. Zeilinger, Rev. Mod. Phys. 71, S288-S297 (1999).

[21] D. Kahneman, J. Beatty, and I. Pollack, Science 157, 218-219 (1967).

[22] J. Silvanto, A. Cowey, N. Lavie, and V. Walsh, Nat. Neurosci. 8, 143-144 (2005).

[23] K. Popper, Logik der Forschung (1935), English edition, Chap. 1 (Routledge, London and New York, 1992).

[24] Y. Kim et al., Phys. Rev. Lett. 84, 1-5 (2000).

[25] M. O. Scully and K. Drühl, Phys. Rev. A 25, 2208-2213 (1982).

[26] N. Brunner, C. Branciard, and N. Gisin, Phys. Rev. A 78, 052110 (2008).

[27] F. H. Thaheld, BioSystems 92, 114-116 (2008).

[28] M. Caponigro, X. Jiang, R. Prakash, and R. Vimal, NeuroQuantology 8, 378-389 (2010).

[29] J. Lachter, F. Durgin, and T. Washington, Vis. Cogn. 7, 269-279 (2000).

[30] J. E. Raymond, K. L. Shapiro, and K. M. Arnell, J. Exp. Psychol. Hum. Percept. Perform. 18, 849-860 (1992).

[31] R. A. Rensink, J. K. O’Regan, and J. J. Clark, Psychol. Sci. 8, 368-373 (1997).

[32] A. M. Treisman and G. Gelade, Cogn. Psychol. 12, 97-136 (1980). 
[33] X. Y. Zou, L. J. Wang, and L. Mandel, Phys. Rev. Lett. 67, 318-321 (1991).

[34] U. Eichmann et al., Phys. Rev. Lett. 70, 2359-2362 (1993).

[35] S. Dürr, T. Nonn, and G. Rempe, Nature 395, 33-37 (1998).

[36] M. Lockwood, Br. J. Philos. Sci. 47, 159-188 (1996).

[37] M. B. Mensky, Opt. Spectrosc. 103, 461-467 (2007).

[38] H. Atmanspacher, Quantum Approaches to Consciousness, in: The Stanford Encyclopedia of Philosophy, edited by E. N. Zalta (2011), http://plato.stanford.edu/entries/qt-consciousness. 